\title{
Incidence of Newly HIV Diagnosed Cases among Foreign Migrants in Italy: 2006-2013
}

Laura Camoni $^{1 *}$, Mariangela Raimondo ${ }^{1}$, Vincenza Regine ${ }^{1}$, Maria Cristina Salfa', Barbara Suligoi' and Referents of HIV Surveillance System ${ }^{2}$ ${ }^{1}$ Centro Operativo AIDS, Dipartimento di Malattie Infettive Parassitarie e Immunomediate, Istituto Superiore di Sanità, viale Regina Elena 299 Rome, Italy ${ }^{2}$ Referents of HIV Surveillance System: Manuela Di Giacomo, Viviana Faggioni (Abruzzo) Francesco Locuratolo, Gabriella Cauzillo (Basilicata), Anna Domenica Mignuoli, Daniele Chirico (Calabria), Guglielmo Borgia (Campania), Alba Carola Finarelli, Erika Massimiliani (Emilia-Romagna), Tolinda Gallo, Cinzia Braida (Friuli-Venezia Giulia), Amalia Vitagliano, Francesco Chini, Paola Barni (Lazio), Giancarlo Icardi, Piero Luigi Lai (Liguria), Maria Gramegna, Liliana Coppola, Alessandra Piatti, Annamaria Rosa (Lombardia), Fabio Filippetti (Marche), Paola Sabatini (Molise), Chiara Pasqualini (Piemonte), Peter Mian, Oswald Moling (PA Bolzano), Danila Bassetti (PA Trento), Maria Chironna, Michele Quarto (Puglia), Stefano Ledda (Sardegna), Gabriella Dardanoni (Sicilia), Fabio Voller, Monica Da Frè, Monia Puglia (Toscana), Anna Tosti, Rita Papili (Umbria), Marina Giulia Verardo, Mauro Ruffier, Echarlod Elisa Francesca (Valle d'Aosta), Francesca Russo, Filippo da Re (Veneto)

\begin{abstract}
Objective: In Europe, during the last years the proportion of foreign migrants among AIDS and HIV cases is growing. In Italy, the number of new cases of HIV represented by foreign migrants has progressively increased, but the new HIV diagnoses incidence among foreign migrants has decreased, because of the increase in the total number of foreign migrants in recent years. Nevertheless, the new HIV diagnoses incidence among foreign migrants is still high if compared to that among Italians. The aim of the study was to analyze the characteristics of foreign migrants with a new HIV diagnosis and the new HIV diagnoses incidence among foreign migrants diagnosed in Italy between 2006 and 2013.

Methods: The new diagnoses incidence was calculated as the ratio between the number of new HIV diagnoses among foreign migrants and the number of foreign migrants residing in Italy. The new HIV diagnoses incidence was compared with that among Italians using the standardized incidence ratio (SIR) adjusted by age and gender.

Results: During the study period (2006-2013), of the 25,545 new diagnoses reported by the HIV surveillance system, $28.8 \%$ were among foreign migrants. The incidence of new HIV diagnoses among foreign migrants for the entire study period was 26.7 cases per 100,000 foreign migrant residents, compared to 5.1 per 100,000 among Italians. The incidence of new HIV diagnoses decreased over time for foreign migrants: from 42.5 per 100,000 foreign migrant residents in 2006 to 20.0 per 100,000 foreign migrant residents in 2013, whereas for Italians it remained stable. The SIR confirmed the marked difference between the two populations: specifically the overall incidence of new diagnoses was more than four times higher among foreign migrants, compared to Italians.
\end{abstract}

Conclusion: The results stress the need to reinforce the efforts aimed at reducing the circulation of HIV in this population.

Keywords: Foreign migrants; Epidemiology; HIV; AIDS

\section{Introduction}

Migrant populations represent a considerable and growing proportion of cases of both AIDS and HIV infection reported in Europe during the last years [1]. Migrants are particularly vulnerable to infection due to numerous social, economic, cultural, and legal factors [1]. These factors include origin from countries that are endemic for HIV, predominance of younger and sexually active persons, rupturing of family ties and couples, in addition to exclusion from society, and language, cultural and socioeconomic barriers [2]. The status of migrant influences the access to prevention and care services and concurs to increase the burden of HIV infection among this population.

In Italy, the number of new cases of HIV among foreign migrants has progressively increased, but the new HIV diagnoses incidence among foreign migrants has decreased, because of the increase in the total number of foreign migrants in recent years $[3,4]$. Nevertheless, the new HIV diagnoses incidence among foreign migrants is still high if compared to that among Italians, suggesting that foreign migrants constitute a group with a high circulation of HIV [5]

More than $70 \%$ of Italy's foreign migrants are from continents where the prevalence of HIV infection is very high (43.5\% from Africa and 21.5\% from South America, in 2013) [4], and there has been an increase in the number of foreign migrants from Eastern Europe, where the occurrence of HIV infection is increasing [1].
The objective of the present study was to estimate the incidence of new diagnoses of HIV infection among foreign migrants in Italy and to describe the demographic and clinical characteristics of this population. We also compared the foreign migrants and the Italian population in terms of trends in HIV new diagnosis incidence over time.

\section{Materials and Methods}

We used individual data on new HIV diagnoses reported in Italy from 2006 to 2013 to the HIV surveillance system. The HIV surveillance system is coordinated by the National AIDS Unit of the National Institute of Health (Rome) and was established in 2008 by a decree of the Ministry of Health [1] which included HIV infection to

*Corresponding author: Dr. Laura Camoni Centro Operativo AIDS, Dipartimento di Malattie Infettive Parassitarie e Immunomediate, Istituto Superiore di Sanità, viale Regina Elena 299 Rome, Italy, Tel: 0649902273; Fax: 0649902755; E-mail: laura.camoni@iss.it

Received April 24, 2015; Accepted May 27, 2015; Published June 10, 2015

Citation: Camoni L, Raimondo M, Regine V, Salfa MC, Suligoi B, et al. (2015) HIV Incidence Estimate among Migrants in Italy: 2006-2013 Incidence of Newly HIV Diagnosed Cases among Foreign Migrants in Italy: 2006-2013. J AIDS Clin Res 6 : 470. doi:10.4172/2155-6113.1000470

Copyright: ( 2015 Camoni L, et al. This is an open-access article distributed unde the terms of the Creative Commons Attribution License, which permits unrestricted use, distribution, and reproduction in any medium, provided the original author and source are credited. 
the list of mandatory notifiable infectious diseases. The notification of new HIV diagnoses were sent from the Infectious diseases clinics to the Italian Regions and then to National AIDS Unit who collect the new diagnoses from the whole country.

For every HIV case, the following individual information are recorded: socio-demographic, clinical, virological and immunological characteristics, date of first HIV positive test, transmission mode (defined to be mutually exclusive), CD4 count at HIV diagnosis, reasons for undergoing testing.

We defined as "foreign migrants" those persons born outside of Italy or with a foreign nationality or citizenship.

Persons with CD $4 \leq 350$ cells/ $\mu \mathrm{L}$ or diagnosed with AIDS (regardless of the CD4 cell count) were defined as late presenters (LP) [2].

The new HIV diagnosis incidence was calculated using as the numerator the annual number of new diagnoses of HIV infection among foreign migrants for the period between 2006 and 2013, and as denominator the annual number of foreign migrants residing in the same period in the same areas. Data on residents for both Italians and foreign migrants were obtained from the National Institute of Statistics (ISTAT) [3].

The comparison of the incidence of new diagnoses among foreign migrants to that among Italians was performed by calculating the standardized incidence ratio (SIR) by age and gender. As standard population, we used the resident Italian population in the study period.

A multivariate logistic regression model was used to identify factors associated with being a foreign migrant with a new HIV diagnosis. Variables with a P-value of 0.05 or less were entered in the model. Specifically we included age, gender, area of residence, HIV transmission mode and late presenters. The statistical analysis was conducted using IBM SPSS 22.0.

\section{Results}

During the study period (2006-2013), of the 25,545 new HIV diagnoses reported by the surveillance system, 7,371 (28.8\%) were among foreign migrants. The number of new HIV diagnoses among foreign migrants increased from 672 in 2006 to 865 in 2013, accounting, respectively, for $33.2 \%$ and $24.0 \%$ of all new HIV diagnoses reported in Italy. In the same period, the number of resident foreign migrants increased, from 1,355,510 in 2006 to 4,325,528 in 2013 (Figure 1).

The incidence of new HIV diagnoses among foreign migrants for the entire study period was 26.7 cases per 100,000 foreign migrants resident, compared to 5.1 per 100,000 Italian residents. The incidence of new diagnoses decreased over time for foreign migrants (Figure 2), from 42.5 diagnoses per 100,000 foreign migrants resident in 2006 to 20.0 diagnoses per 100,000 foreign migrants resident in 2013, whereas for Italian residents remained stable (5.3 per 100,000 Italian residents in 2006 and 4.9 per 100,000 Italian residents in 2013).

The SIR confirmed the marked difference between the two populations, with the overall incidence of new HIV diagnoses among foreign migrants being four times greater than that among Italians (Table 1).

This difference decreased over time; in fact, in 2006 the incidence of new diagnoses was about six times higher among foreign migrants, whereas it was about three times higher in 2013, as compared to Italians.

Of the HIV seropositive foreign migrants, $54.9 \%$ were males; the median age at diagnosis was 33 years (Interquartile range - IQR 28-40), and it significantly different by gender (35 years (IQR 29-41) for males and 31 years (IQR 26-38) for females). Three-thousand-three-hundredeighty-two (45.9\%) were notified in Northern Italy, 2,300 (31.2\%) in Central Italy and 1,689 (22.9\%) in Southern Italy (data not show).

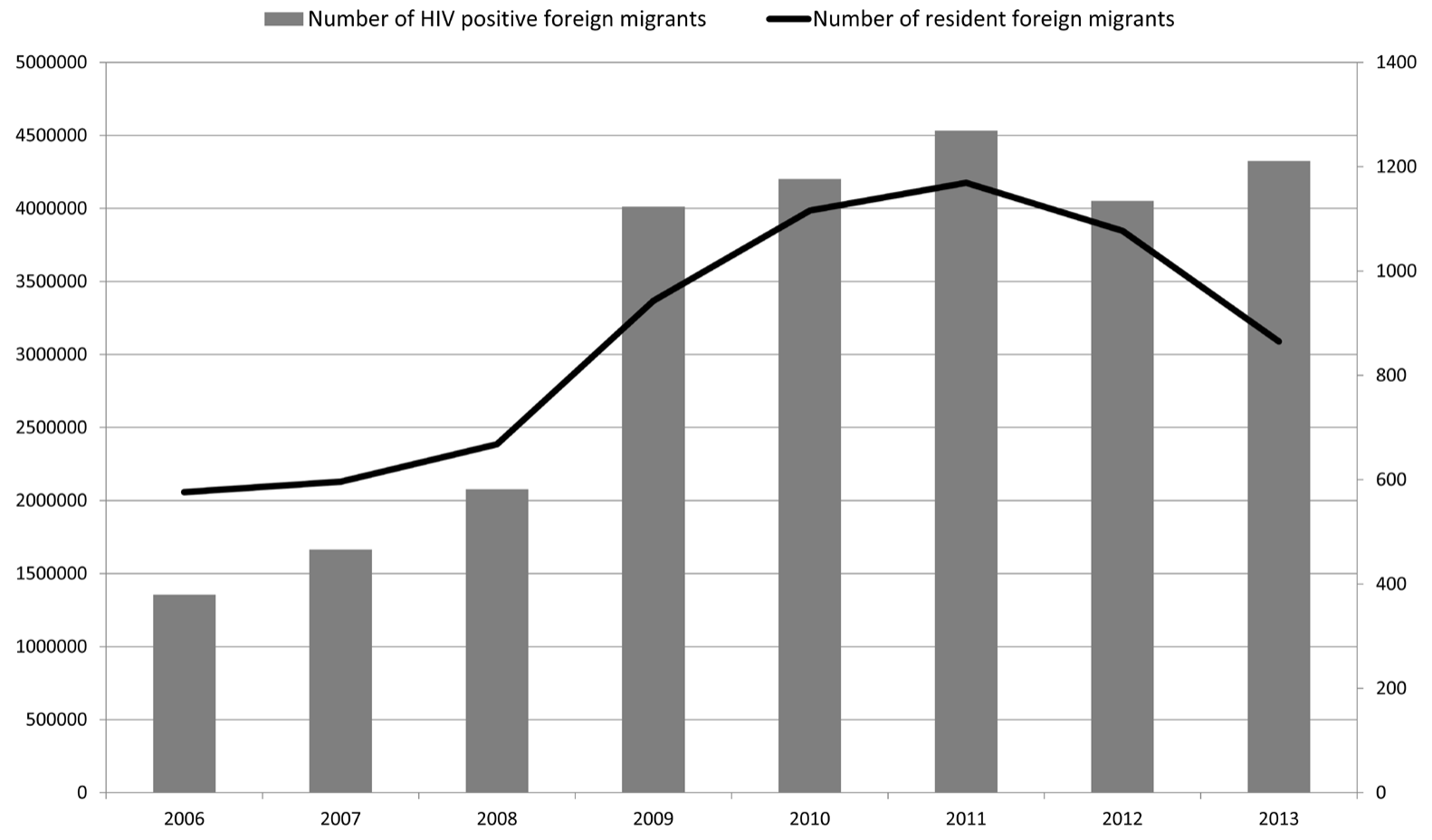

Figure 1: Number of resident foreign migrants and new HIV diagnoses among foreign migrants in Italy (2006- 2013). 


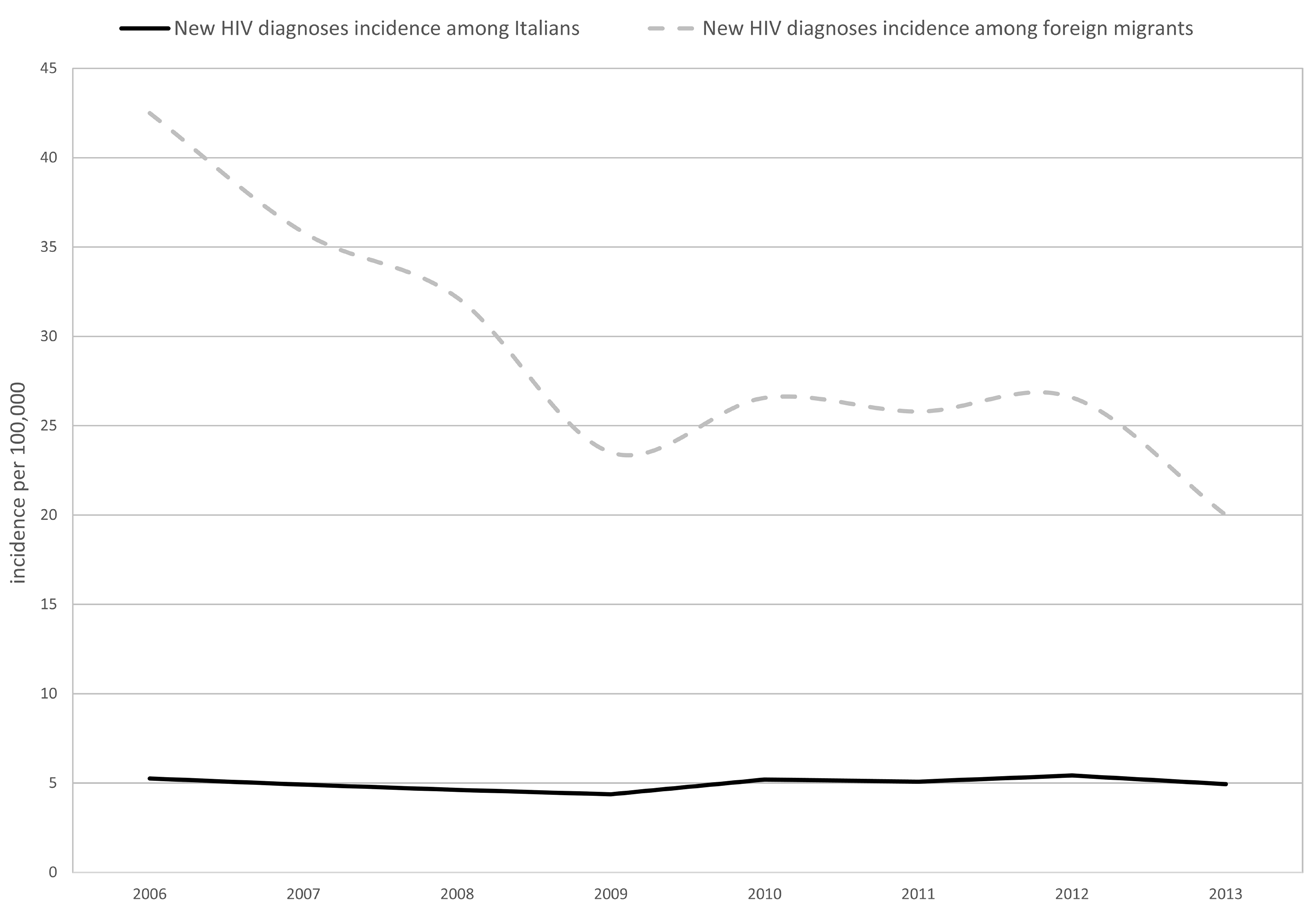

Figure 2: New HIV diagnoses incidence among foreign migrants and Italians (per 100,000).

\begin{tabular}{|c|c|c|c|c|}
\hline Year & $\begin{array}{l}\text { Incidence among } \\
\text { Italians }(x \quad 100,000)\end{array}$ & $\begin{array}{l}\text { Incidence among } \\
\text { foreign migrants } \\
\quad(\times 100,000)\end{array}$ & $\begin{array}{l}\text { Standardized incidence among foreign } \\
\text { migrants } \\
(\times 100,000)^{*}\end{array}$ & Standardized incidence ratio \\
\hline 2006 & 5,3 & 42,5 & 34,5 & 6,6 \\
\hline 2007 & 4,9 & 35,8 & 27,8 & 5,7 \\
\hline 2008 & 4,6 & 32,2 & 24,3 & 5,3 \\
\hline 2009 & 4,4 & 23,5 & 19,1 & 4,4 \\
\hline 2010 & 5,2 & 26,6 & 21,1 & 4,1 \\
\hline 2011 & 5,1 & 25,8 & 20,0 & 3,9 \\
\hline 2012 & 5,4 & 26,6 & 21,1 & 3,9 \\
\hline 2013 & 4,9 & 20,0 & 15,9 & 3,2 \\
\hline
\end{tabular}

*Standardized by age and gender

Table 1: Crude and standardized incidence of new HIV diagnoses among Italians and foreign migrants, 2006-2013.

Among foreign migrants with a new diagnosis of HIV infection, $46.6 \%$ were from Africa (primarily from Nigeria, Ghana and Ivory Coast), 24.3\% were from European countries (primarily from Romania and Ukraine), $18.4 \%$ were from South America (Brazil and Peru), $4.3 \%$ from Asia, and $6.4 \%$ was undetermined. The distribution of new HIV diagnoses by area of origin changed over time: in 2006, persons from Africa represented $45.1 \%$ of the new diagnoses among foreign migrants, whereas in 2013 they represented $42.8 \%$; the proportion of persons from European countries increased from $25.6 \%$ in 2006 to $31.0 \%$ in 2013; the proportion of persons from Latin America increased from $12.0 \%$ in 2006 to $18.4 \%$ in 2013 ; finally the proportion represented by persons from Asia remained quite stable (3.6 in 2006 and 4.3 in 2013) (data not shown).

The HIV transmission mode was heterosexual for $64.6 \%$ of the seropositive foreign migrants for the overall study period; the proportion of foreign migrants with this exposure category slightly increased over time, from $60.3 \%$ in 2006 to $61.5 \%$ in 2013. In the meantime, the proportion of MSM increased from 14.5\% in 2006 to
$18.7 \%$ in 2013, the proportion of IDU increased from 2.5\% in 2006 to $3.3 \%$ in 2013 and the proportion of other transmission mode decreased from $11.2 \%$ in 2006 to 9.5 in 2013.

Among persons from European countries and from Africa heterosexuals accounted for $55.5 \%$ and $78.6 \%$, respectively, whereas among South Americans and Italians the majority was MSM or heterosexuals (South America: 45.8\% MSM and 40.1\% heterosexuals; Italy: $39.7 \%$ MSM and $38.4 \%$ heterosexuals) (Figure 3).

Among foreign migrants with a new diagnosis of HIV infection, $39.7 \%$ were late presenters; the proportion of foreign migrants late presenters increased from $29.8 \%$ in 2006 to $40.1 \%$ in 2013 (data not shown).

At the multivariate analysis, factors significantly associated to being a foreign migrant with HIV infection were being a females; having a younger age; being residents in the Centre of Italy; being heterosexuals; being late presenters and being diagnosed between 2006-2008 (Table 2). 


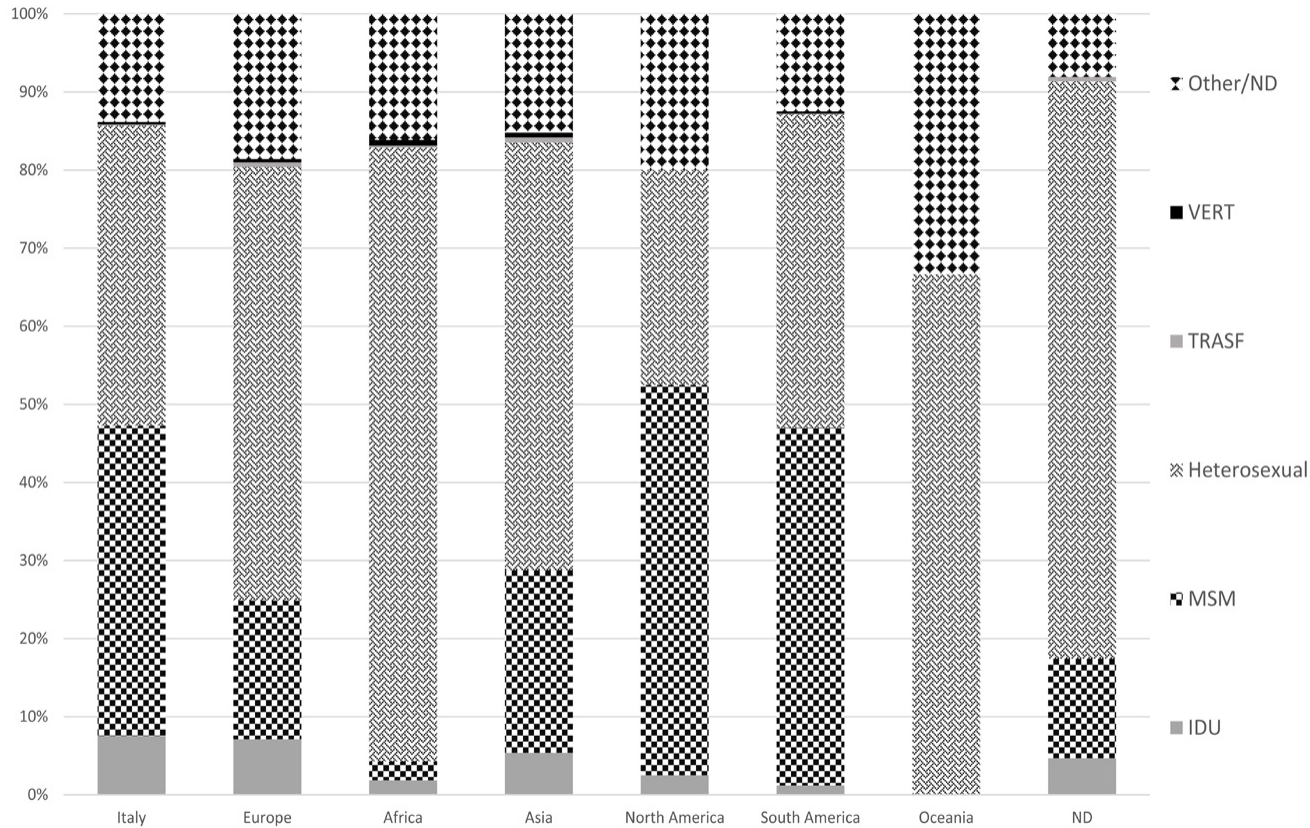

Figure 3: Distribution of new HIV diagnoses among foreign migrants, by area of origin and mode of transmission (2006-2013).

A higher proportion of foreign migrants, compared to Italians, underwent HIV testing on occasion of a pregnancy test $(72.3 \%$ vs 27.7\%; p-value $=0.000$ ). On the opposite, underwent HIV testing because of complaining of clinical conditions associated with HIV infection, having a partner recently found HIV-positive, screening for other diseases, having a partner HIV-unknown, having other parenteral blood exposures, medical routine check and having engaged in sexual risk behavior, were not perceived as relevant reasons for undergoing HIV testing from foreign migrants compared to Italians (Figure 4).

\section{Discussion}

Since 2006, the number of foreign migrants with a new diagnosis of HIV infection increased. This increased can be partly explained by a higher migratory flow in these last years; particularly in 2008 the number of resident foreign migrants grew greatly as a consequence of an Italian Decree regularizing the number of foreign migrants who can enter in Italy [4].

The incidence standardized by age and gender used to minimize age-and gender-related differences (foreign migrants are generally younger than Italians and prevalently male) $[5,6]$ confirmed that the incidence of new HIV diagnoses was consistently higher among foreign migrants, compared to Italians, throughout the study period. This suggests that, despite the fact that foreign migrants are generally younger and healthier [7-9] they constitute a population with a high circulation of HIV infection. However, we were not able to establish whether the infection was acquired in Italy. The finding of a high percentage of infected foreign migrants from geographic areas at high HIV prevalence (Africa, Eastern Europe and Asia) could suggest that these persons acquired the infection in their country of origin and that they were already infected when entering Italy, although further investigation will be necessary to evaluate this hypothesis.

The new HIV diagnoses among foreign migrants were concentrated among young persons and heterosexuals mainly from countries with a high circulation of HIV (such as Africa and South America) as reported in other EU countries [10] emphasizing the necessity to acknowledge the socio-demografic diversity of foreign migrants living with HIV.

In Italy, foreign migrants with HIV are geographically concentrated in some areas of the country, especially in the Centre and in the South of Italy. This finding can reflect a poor access to the Italian health services for foreign migrants or HIV testing in these areas.-

Foreign migrants are more likely to be diagnosed late for HIV than those born in Italy pointing out that the promotion of earlier diagnosis is a major topic both in Italy [9,11] and in other European Countries [5].

A medical control during pregnancy was the main reason for HIV testing among foreign migrants suggesting a low perception of having engaged in at-risk behaviors, underlying the importance of take advantage of the pregnancy to identify female foreign migrants with HIV and the need of action in pregnancy-specific HIV prevention and treatment policy.

In interpreting the results of this study, some limitations need to be considered. First, the date of infection was not available. In fact, the estimated incidence rates are not presented as the true incidence of new HIV infections but rather as the incidence of new diagnoses. The time that elapses between acquiring HIV infection and diagnosis can vary, and it depends on the individual's perception of risk and on access to testing. Nonetheless, we analyzed temporal trends in new HIV diagnoses incidence, and it can be reasonably assumed that this time difference remains, on average, constant over the years, without greatly affecting the observed incidence curve.

Another limitation is that foreign migrants may be less likely to undergo HIV testing because they are not aware of or familiar with the available healthcare services, because of language difficulties, or because they are not legally present in Italy and fear that they will be reported to the authorities. Thus, the estimated new HIV diagnoses incidence among foreign migrants may represent an underestimate. 
Citation: Camoni L, Raimondo M, Regine V, Salfa MC, Suligoi B, et al. (2015) HIV Incidence Estimate among Migrants in Italy: 2006-2013 Incidence of Newly HIV Diagnosed Cases among Foreign Migrants in Italy: 2006-2013. J AIDS Clin Res 6: 470. doi:10.4172/2155-6113.1000470

Page 5 of 6

\begin{tabular}{|c|c|c|c|c|c|c|c|}
\hline & italians & $\%$ & migrants & $\%$ & $p$ value & OR & $(95 \% \mathrm{Cl})$ \\
\hline Total & 18174 & & 7371 & & & & \\
\hline Gender* & & & & & 0.000 & & \\
\hline Male & 15269 & 84.0 & 4048 & 54.9 & & 1 & \\
\hline Female & 2902 & 16.0 & 3322 & 45.1 & & 2.51 & $(2.34-2.70)$ \\
\hline missing & 3 & 0.0 & 1 & 0.0 & & & \\
\hline Median age at diagnosis in years ** (IQR) & $39(32-48)$ & & $33(28-40)$ & & 0.000 & & \\
\hline Age class at diagnosis (in years) ${ }^{*}$ & & & & & 0.000 & & \\
\hline$<=24$ & 1279 & 7.0 & 966 & 13.1 & & 1 & \\
\hline $25-34$ & 4877 & 26.8 & 3129 & 42.5 & & 0.89 & $(0.80-0.99)$ \\
\hline $35-44$ & 5959 & 32.8 & 2275 & 30.9 & & 0.53 & $(0.47-0.59)$ \\
\hline $45-54$ & 3733 & 20.5 & 805 & 10.9 & & 0.27 & $(0.24-0.30)$ \\
\hline$>=55$ & 2318 & 12.8 & 194 & 2.6 & & 0.09 & $(0.07-0.11)$ \\
\hline missing & 8 & 0.0 & 2 & 0.0 & & & \\
\hline Area of residence ${ }^{*}$ & & & & & 0.000 & & \\
\hline Northern Italy & 10109 & 55.6 & 3382 & 45.9 & & 1 & \\
\hline Centre Italy & 4258 & 23.4 & 2300 & 31.2 & & 1.71 & $(1.59-1.84)$ \\
\hline Southern Italy & 3807 & 20.9 & 1689 & 22.9 & & 1.29 & $(1.19-1.39)$ \\
\hline HIV transmission mode* & & & & & 0.000 & & \\
\hline IDU & 1368 & 7.5 & 241 & 3.3 & & 1 & \\
\hline MSM & 7221 & 39.7 & 1159 & 15.7 & & 1.04 & $(0.89-1.22)$ \\
\hline Heterosexual & 6984 & 38.4 & 4765 & 64.6 & & 3.45 & $(2.97-3.99)$ \\
\hline Other & 84 & 0.5 & 64 & 0.9 & & 2.15 & $(1.47-3.15)$ \\
\hline Not determined & 2517 & 13.8 & 1142 & 15.5 & & 2.58 & $(2.20-3.03)$ \\
\hline Late presenters* & & & & & 0.000 & & \\
\hline No & 10810 & 60.3 & 4448 & 60.3 & & 1 & \\
\hline Yes & 7364 & 39.7 & 2923 & 39.7 & & 1.19 & $(1.11-1.27)$ \\
\hline Years of diagnosis* & & & & & 0.000 & & \\
\hline $2006-2008$ & 6927 & 38.1 & 3144 & 42.7 & & 1 & \\
\hline $2009-2013$ & 11247 & 61.9 & 4227 & 57.3 & & 0.90 & $(0.84-0.96)$ \\
\hline
\end{tabular}

${ }^{*}$ Chi square test

${ }^{* *} \mathrm{U}$ Mann Whitney test

Table 2: Characteristics of new HIV diagnoses among Italians and foreign migrants.

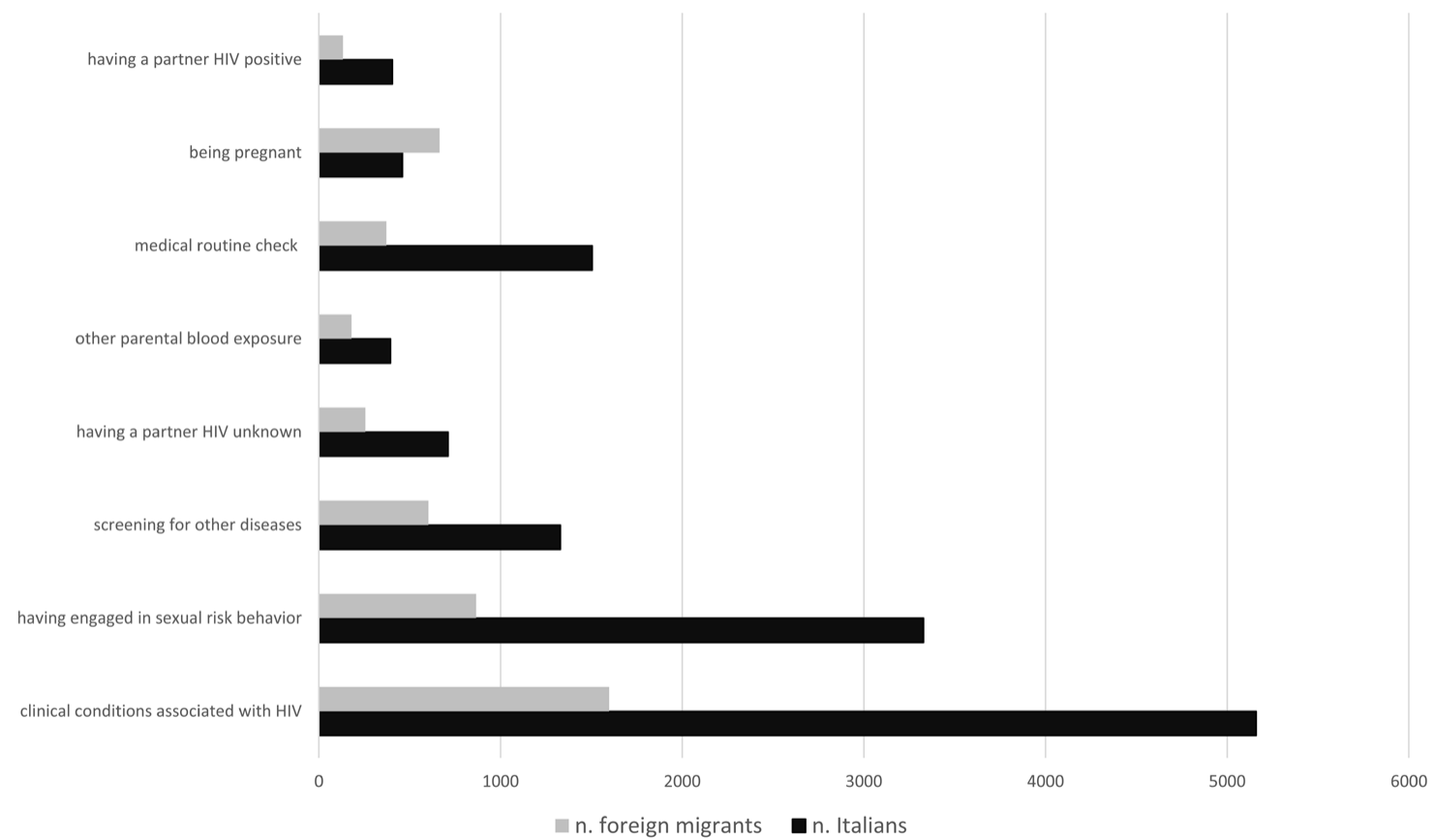

Figure 4: Reasons for HIV testing among Italians and foreign migrants (2006-2013). 
Citation: Camoni L, Raimondo M, Regine V, Salfa MC, Suligoi B, et al. (2015) HIV Incidence Estimate among Migrants in Italy: 2006-2013 Incidence of Newly HIV Diagnosed Cases among Foreign Migrants in Italy: 2006-2013. J AIDS Clin Res 6: 470. doi:10.4172/2155-6113.1000470

Page 6 of 6

It must also be considered that the denominator used in calculating the incidence only consisted of those foreign migrants legally present, given that, based on the official data, it is not possible to determine the number of undocumented foreign migrants. According to Caritas, in 2012 there were an estimated 5,186,000 foreign migrants in Italy (including those illegally present that are living in Italy without any documents), which is approximately $21 \%$ more than the number based on resident foreign migrants provided by ISTAT [3]. If we were to use the total number of foreign migrants as the denominator, and assuming that there are no HIV infected persons among foreign migrants without a residence permit, the 2012 new HIV diagnoses incidence would decrease from 22.3 to 17.4 per 100,000 foreign migrants. Nonetheless, this estimate is still three times higher than that among Italians.

In conclusion, the results indicate that although the incidence of newly HIV diagnosed cases among foreign migrants seems to have decreased in the past 6 years, it is still high if compared to that among Italians, suggesting that foreign migrants constitute a population subgroup with a high circulation of HIV. Foreign migrants should be an important population in the development of preventive and clinical strategies built to promote early diagnosis and easy access to care.

\section{References}

1. Italia, Decreto Ministeriale 31 marzo (2008) Istituzione del Sistema di Sorveglianza delle nuove diagnosi di infezione da HIV. Gazzetta Ufficiale n.

\section{5, 28 luglio.}

2. Antinori A, Coenen T, Costagiola D, Dedes N, Ellefson M, et al. (2011) Late presentation of HIV infection: a consensus definition. HIV Med 12: 61-64.

3. ISTAT (2015) Demografia in cifre.

4. Circolare Ministero Interno - Decreto flussi 2008

5. European Centre for Disease Prevention and control (ECDC) (2013) ECDC Meeting Report. ECDC Workshop: improving the monitoring of HIV among migrant populations in Europe.

6. Ufficio Nazionale Antidiscriminazione Razziali (UNAR) Dossier statistico 2013 Centro Studi e Ricerche IDOS/Immigrazione, 2013.

7. Geraci S (2000) [Sexually transmitted diseases among immigrants in Italy]. See comment in PubMed Commons below Ann Ist Super Sanita 36: 445-449.

8. Marceca M, Geraci S, Ardigò M (2007) Esperienza migratoria, salute e disuguaglianze. Atti IX Convegno. Fragilità sociale e tutela della salute: dalle disuguaglianze alla corresponsabilità. Rapporti ISTISAN, 07/14.

9. Saracino A, Tartaglia A, Trillo G, Muschitiello C, Bellacosa C, et al. (2014) Late presentation and loss to follow-up of immigrants newly diagnosed with HIV in the HAART era. J Immigr Minor Health 16: 751-755.

10. European Centre for Disease Prevention and control (ECDC) Technical Report (2010) Migrant health: epidemiology of HIV and AIDS in migrant communities and ethnic minorities in EU/EEA countries. ECDC, 2009.

11. Sulis G, El Hamad I, Fabiani M, Rusconi F, Maggiolo F, et al. (2014) Clinical and epidemiological features of HIVIAIDS infection among migrants at first access to healthcare services as compared to Italian patients in Italy: a retrospective multicenter study, 2000-2010. Infection 42: 848-867. 